\title{
Embolic retinopathy due to Corynebacterium minutissimum endocarditis
}

\author{
BRIAN J HERSCHORN AND ALEXANDER J BRUCKER
}

From the Department of Ophthalmology, University of Pennsylvania School of Medicine, and the Scheie Eye Institute, Philadelphia, Pennsylvania 19104, USA

SUMMARY Infective embolic retinopathy as a sequela of bacterial endocarditis is described in a 31year-old woman with mitral valve prolapse. The infective organism, Corynebacterium minutissimum, has not been previously found to cause ocular or multisystem diseases. It is a common mucocutaneous inhabitant which causes erythrasma. In our case report both ocular involvement and septicaemia were present. The infection was confirmed by positive serial blood cultures. Mitral valve prolapse was confirmed by echocardiography. On clinical examination the retinopathy consisted of white intraretinal lesions which resolved with antibiotic therapy. By fluorescein angiography focal areas of hypofluorescence corresponding to the white fundus lesions were present. Optic disc oedema was also seen.

Corynebacterium minutissimum is a common mucocutaneous inhabitant which causes erythrasma, a mild, localised, superficial skin infection. ${ }^{2}$ This communication reports an unusual case of embolic retinopathy, bacterial endocarditis, mitral valve prolapse, and septicaemia due to $C$. minutissimum. The species has not previously been implicated as the cause of ocular or multisystem infection.

Sterile emboli causing cerebral ischaemia ${ }^{3}$ and retinal vascular occlusions ${ }^{4}$ have been shown to have their origin from prolapsing mitral valves. In the present case vegetation on the prolapsing mitral valves is believed to be the source of the emboli.

\section{Case report}

A 31-year-old woman was admitted to the Scheie Eye Institute one day after the onset of decreased vision and photophobia of the left eye. During the preceding week she noted metamorphopsia and spots in that eye. For the last four months she had had fever and chills, frontal headaches, anorexia, weight loss, fatigue, intermittent migratory polyarthritis, and arthralgia. She had been placed on prophylactic antibiotics after rheumatic fever at the age of 7 years; this medication ceased three years prior to admission to hospital. After tubal ligation one year before

Correspondence to Alcxander J Brucker, MD, Schcic Eyc Institute, 51 North 39th Strect, Philadelphia, Pennsylvania 19104, USA. admission echocardiography had shown prolapse of the mitral valve. In addition she had recently undergone dental surgery.

Visual acuity was $6 / 6$ in each eye. Slit-lamp biomicroscopy showed $1+$ flare and cells in the anterior chamber and vitreous bilaterally. Intraocular pressures were normal. Ophthalmoscopic examination of the right eye showed one white intraretinal lesion with a fine rim of haemorrhage one disc

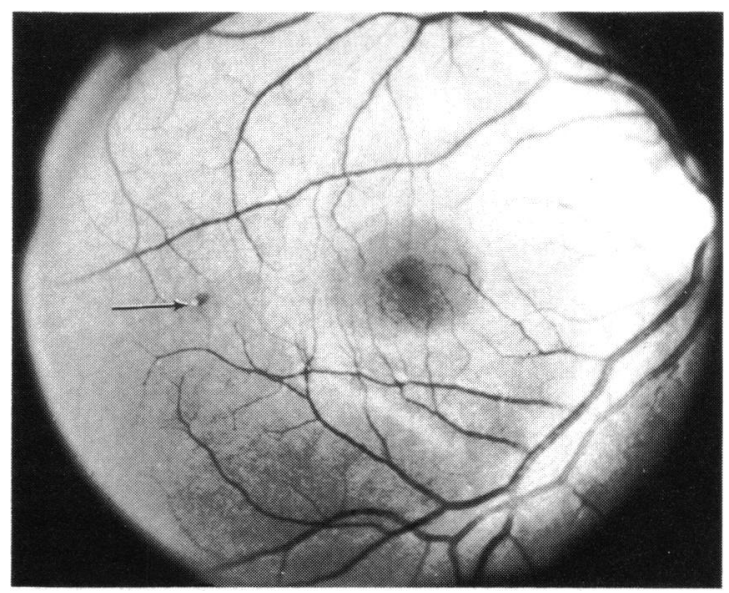

Fig. 1 Right macula. White intraretinal lesion with rim of haemorrhage prior to therapy (arrow). 


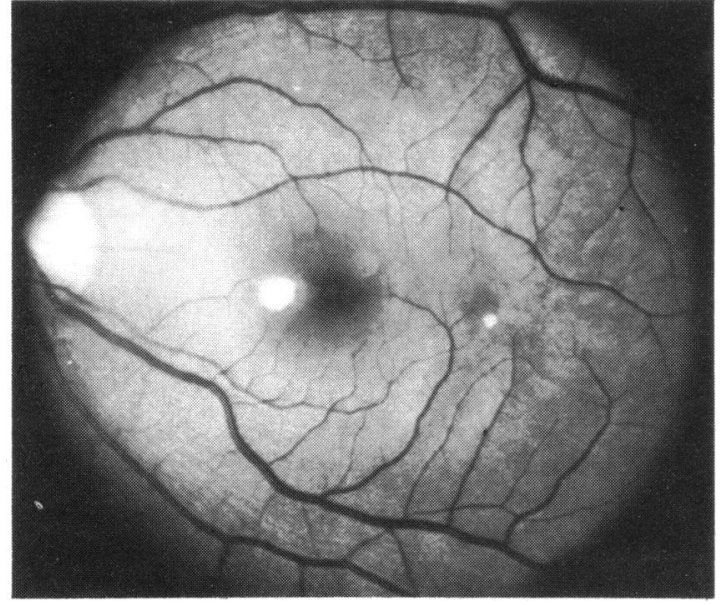

Fig. 2 Left macula. Discrete white intraretinal lesions seen prior to therapy. (Photographic artefact superotemporal to the fovea).

diameter temporal to the fovea (Fig. 1). A second white intraretinal lesion was seen near the ora serrata. In the left eye a white intraretinal lesion of one-fifth disc diameter was seen in the papillomacular bundle (Fig. 2). A zone of surrounding oedema extended to the nasal edge of the fovea. A second lesion was situated one and one half disc diameters temporal to the fovea (Fig. 2), with a third lesion located peripherally. Fluorescein angiography (Fig. 3) showed areas of hypofluorescence corresponding to the white lesions bilaterally. Fluorescein dye from the surrounding retinal capillaries filled

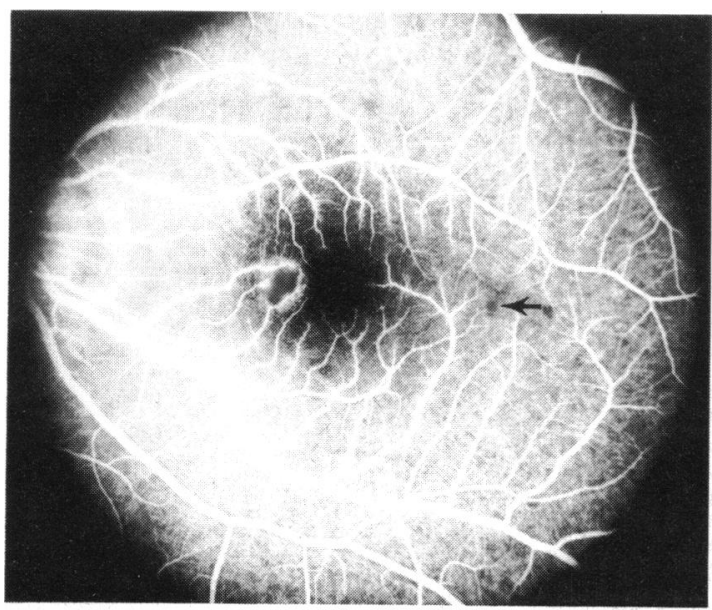

Fig. 3A Left macula. Fluorescein angiogram prior to therapy. Midvenous phase showing area of hypofluorescence corresponding to large white lesion seen clinically (Fig. 2). Smaller lesion (arrow) also appears hypofluorescent. these areas and showed late staining of the retina. Leakage of dye from both the optic discs consistent with papillitis was seen (Fig. 4). Cardiac auscultation revealed a grade II/VI apical, holosystolic, nonradiating murmur. The rest of the examination, including mucocutaneous tissues, was entirely normal.

Blood cultures were positive for significant exclusive growth of Corynebacterium minutissimum. The organism was sensitive to penicillin. Echocardiography indicated vegetations on the anterior leaflet of a prolapsing mitral valve consistent with bacterial endocarditis. Blood studies revealed a microcytic, hypochromic iron deficiency anaemia. Serum IgM and IgG were elevated at $2451 \mathrm{mg} / \mathrm{dl}$ and $672 \mathrm{mg} / \mathrm{dl}$ respectively $(24.5$ and $6 \cdot 7 \mathrm{~g} / \mathrm{l})$.

Treatment for subacute bacterial endocarditis was initiated with 4 million units of aqueous penicillin intravenously every four hours for four weeks.

Funduscopic examination during hospital admission showed rapid resolution of the peripheral lesions, while the lesions in the posterior pole resolved slowly. Five weeks after therapy was in itiated the lesion in the right fundus had disappeared and the lesions of the left fundus showed significant improvement of both clinical and fluorescein angiographic appearances.

\section{Discussion}

Corynebacterium minutissimum has not been reported previously as a cause of endophthalmitis and endocarditis. Corynebacteria are frequent inhabitants of normal skin and mucous membranes ${ }^{56}$ and frequently contaminate blood cultures. ${ }^{7} C$.

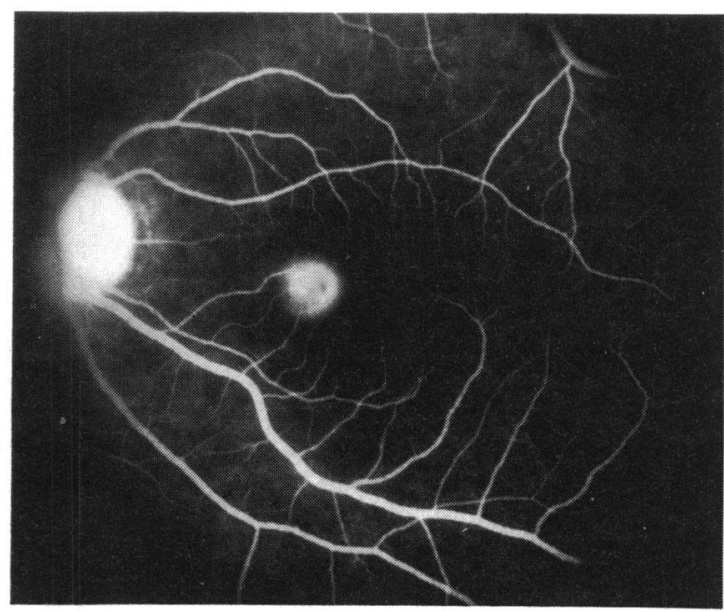

Fig. 3B Late phase. Fluorescein dye fills area of previously described hypofluorescence. Smaller lesion is essentially inapparent. 


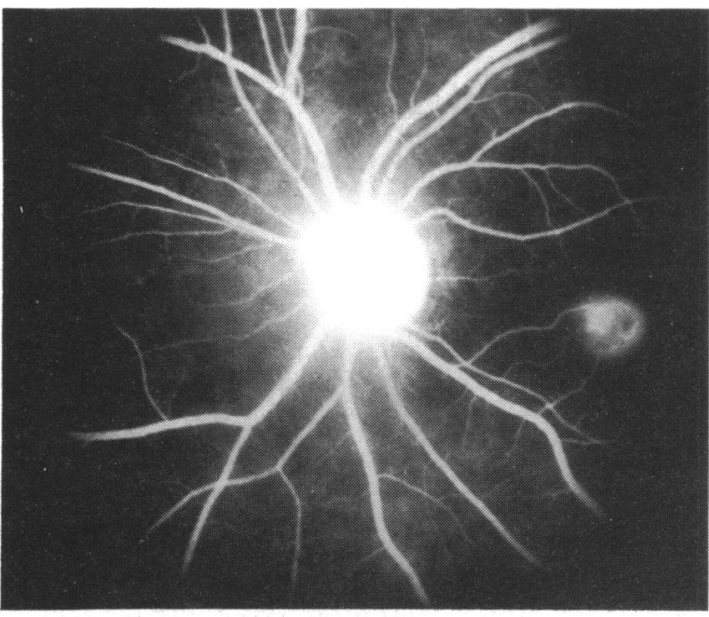

Fig. 4 Left eye. Diffuse leakage of dye from the optic disc late in the angiogram.

minutissimum has been considered to be systemically harmless in humans. It is responsible, however, for erythrasma, a mild, chronic, localised, superficial infection of body clefts and folds. ${ }^{1}$ Cases of corynebacterial endocarditis constitute less than $0.3 \%$ of all cases of endocarditis ${ }^{8}$ and usually affected prosthetic heart valves. None have been reported on an isolated prolapsing mitral valve. ${ }^{6-11}$ Generally, the reported incidence of bacterial endocarditis in mitral valve prolapse is 2 to $6 \%$."

In the present case $C$. minutissimum was confirmed by serial blood cultures. The mitral valve prolapse may have served as a predisposing factor for the infection. The ocular signs consisted exclusively of multiple, intraretinal white lesions sometimes associated with a fine rim of haemorrhage. By fluorescein angiography leakage of dye into the tissue surrounding the lesions suggested an active inflammatory process. Papillitis, which was not clinically apparent, was revealed by the angiographic study. The nature of the lesions is thought to be the accumulation of the inflammatory white cells surrounding the sites of bacterial emboli.

\section{References}

1 Sarkany I, Taplin D, Blank H. The etiology and treatment of erythrasma. J Invest Dermatol 1961; 37: 282-90, 801-3.

2 Hoeprich PD, ed. Infectious diseases, 2nd ed. Hagerstown: Harper and Row, 1977.

3 Barnett HJM, Jones MW, Boughner DR, Kostuk WJ. Cerebral ischemic events associated with prolapsing mitral valves. Arch Neurol 1976; 33: 777-82.

4 Woldoff HS, Gerber M, Desser KB, Benchimol A. Retinal vascular lesions in two patients with prolapsed mitral valve leaflets. Am J Ophthalmol 1975; 79: 382-5.

5 Locatcher-Khorzao D, Segal BC. Microbiology of the eye. St Louis: Mosby, 1972.

6 Kaplan D, Weinstein L. Diphtheroid infections of man. Ann Intern Med 1969; 70: 919-29.

7 Washington JA II. Comparison of two commercially available media for detection of bacteremia. Appl Microbiol 1971; 22: 604-7.

8 Reid JD, Greenwood L. Corynebacterial endocarditis. Arch Intern Med 1967; 119: 106-10.

9 Blount JG. Bacterial endocarditis. Am J Med 1965; 38: 909-22.

10 Gerry JL, Greenbough WB III. Diphtheroid endocarditis: report of nine cases and review of the literature. Johns Hopkins Med J 1976; 139: 61-8.

11 Coker JE, Dworzack DL, Hodges GR, Petric SC, Pugh DM. The infected mitral valve. J Kans Med Soc 1978; 79: 550-2. 